\title{
Warped Discrete-Fourier Transform: Theory and Applications
}

\author{
Anamitra Makur, Senior Member, IEEE and Sanjit K. Mitra, Life Fellow, IEEE
}

\begin{abstract}
In this paper, we advance the concept of warped discrete-Fourier transform (WDFT), which is the evaluation of frequency samples of the $z$-transform of a finite-length sequence at nonuniformly spaced points on the unit circle obtained by a frequency transformation using an allpass warping function. By factorizing the WDFT matrix, we propose an exact computation scheme for finite sequences using less number of operations than a direct computation. We discuss various properties of WDFT and the structure of the factoring matrices. Examples of WDFT for first- and second-order allpass functions is also presented. Applications of WDFT included are spectral analysis, design of tunable FIR filters, and design of perfect reconstruction filterbanks with nonuniformly spaced passbands of filters in the bank. WDFT is efficient to resolve closely spaced sinusoids. Tunable FIR filters may be designed from FIR prototypes using WDFT. In yet another application, warped PR filterbanks are designed using WDFT and are applied for signal compression.
\end{abstract}

Index Terms-Allpass, DFT, frequency warping, warped DFT.

\section{INTRODUCTION}

$\mathbf{T}$ HE discrete-Fourier transform (DFT) is an important tool in digital signal processing, and often finds applications in both analysis and design of discrete-time signals and systems. The $N$-point DFT $X[k], 0 \leq k \leq N-1$, of a length- $N$ sequence $x[n], 0 \leq n \leq N-1$, is given by the frequency samples of the $z$-transform $\bar{X}(z)$ of the sequence $x[n]$ evaluated at $N$ uniformly-spaced points $z=e^{j 2 \pi k / N}, 0 \leq k \leq N-1$, on the unit circle, i.e.,

$$
X[k]=\left.X(z)\right|_{z=e^{j 2 \pi k / N}}=\sum_{n=0}^{N-1} x[n] e^{-j 2 \pi n k / N} .
$$

For spectral analysis applications, the DFT provides a fixedfrequency resolution given by $2 \pi / N$ over the whole frequency range $[0,2 \pi]$.

There are applications where it is desirable to compute the frequency samples of the $z$-transform of a finite-length sequence with an unequal resolution. To this end, Oppenheim and Johnson proposed the transformation of $x[n]$ to a new sequence $y[n]$ by means of an allpass network [1]. The magnitude of the frequency samples of $Y(z)$, the $z$-transform $y[n]$,

\footnotetext{
Manuscript received June 21. 2000; revised February 4. 2001. This work was supported in part by the University of California, in part by Lucent Technologies, in part by the National Semiconductor Corporation, in part by the Tektronix Corporation, in part by the Raytheon Missile Systems, and in part by the Xerox Corporation.

A. Makur is with the Department of Electrical Communication Engineering, Indian Institute of Science, Bangalore 560012, India.

S. K. Mitra is with the Department of Electrical and Computer Engineering, University of California, Santa Barbara, CA 93106 USA.

Publisher Item Identifier S 1057-7122(01)07714-5.
}

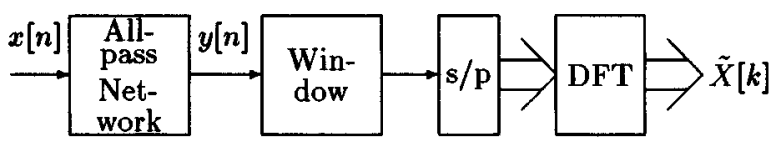

Fig. 1. The scheme for computing the magnitude spectrum with unequal resolution using the FFT.

at equally-spaced points on the unit circle are the magnitudes of the frequency samples of $X(z)$ at unequally spaced points. Since the allpass network has an IIR transfer function, $y[n]$ is an infinite-length sequence and hence, must be made into a finite-length sequence by using an appropriate window function before its DFT can be computed. Fig. 1 shows their scheme. Moreover, the initial conditions of the IIR allpass network will propagate to the output. As a result, this scheme provides an approximate estimate of the magnitudes of the DFT of $x[n]$ at unequal resolutions.

The nonuniform DFT (NDFT) proposed recently [2] is the most general form of DFT that can be employed to evaluate the frequency samples of $X(z)$ at $N$ arbitrary but distinct points in $z$-plane. If $z_{k}, 0 \leq k \leq N-1$, denote $N$ distinct frequency points in the $z$-plane, the $N$-point NDFT of the length- $N$ sequence $x[n]$ is then given by

$$
X_{\mathrm{NDFT}}[k]=X\left(z_{k}\right)=\sum_{n=0}^{N-1} x[n] z_{k}^{-n}, \quad 0 \leq k \leq N-1 .
$$

In matrix form, the above $N$ equations can be written in the form

$$
\left[\begin{array}{c}
X_{\mathrm{NDFT}}[0] \\
X_{\mathrm{NDFT}}[1] \\
\vdots \\
X_{\mathrm{NDFT}}[N-1]
\end{array}\right]=\mathbf{D}_{N} \cdot\left[\begin{array}{c}
x[0] \\
x[1] \\
\vdots \\
x[N-1]
\end{array}\right]
$$

where $\mathbf{D}_{N}$ is the $N \times N$ NDFT matrix given by

$$
\mathbf{D}_{N}=\left[\begin{array}{cccc}
1 & z_{0}^{-1} & \cdots & z_{0}^{-N+1} \\
1 & z_{1}^{-1} & \cdots & z_{1}^{-N+1} \\
\vdots & \vdots & & \vdots \\
1 & z_{N-1}^{-1} & \cdots & z_{N-1}^{-N+1}
\end{array}\right]
$$

which is seen to be a Van der Monde matrix and is invertible if the points $z_{k}$ are distinct. In general, the computation of the NDFT samples involves the multiplication of the NDFT matrix $\mathrm{D}_{N}$ with the length- $N$ vector composed of samples of $x[n]$, and is computationally intensive requiring $N^{2}$ complex multiplications.

The NDFT computation can be made computationally efficient by imposing some restrictions on the locations of the 
points $z_{k}$. For example, if the points $z_{k}$ are located equidistant on the unit circle at $z_{k}=e^{j 2 \pi k / N}$, then the NDFT reduces to the conventional DFT which can be computed very efficiently using fast Fourier transform (FFT) algorithms [3]. Note that the resolution of the DFT depends on the spacing between two consecutive frequency points and an increase in the frequency resolution can be obtained by increasing the DFT length. However, an increase in the DFT length also increases the computational complexity.

In this paper, we consider an alternate structure to the location of the frequency points $z_{k}$ by applying an allpass transformation to warp the frequency axis. Then, uniformly spaced points on the warped frequency axis are equivalent to a nonuniform spacing frequency points on the original frequency axis. This has led to the concept of the warped DFT (WDFT) which evaluates the frequency samples of $X(z)$ at unequally spaced points on the unit circle. By choosing the warping parameter we can space some of the frequency samples closer to each other providing higher resolution in a selected frequency range without increasing the DFT length. We then propose an efficient realization of the WDFT that is exact and easier to implement than the direct WDFT computation. We include a number of applications of the WDFT.

\section{WDFT}

\section{A. Definition, Realization and Properties}

The WDFT is a special case of the more general nonuniform DFT [2]. More specifically, the $N$-point WDFT $\hat{X}[k]$ of a length- $N$ sequence $x[n]$ is given by $N$ equally spaced frequency samples of a modified $z$-transform $X(\hat{z})$ obtained from $X(z)$ by applying the transformation

$$
z^{-1}=B(\hat{z})
$$

where $B(\hat{z})$ is an $M$ th-order real coefficient allpass function. ${ }^{1}$ Note that the allpass transformation warps the frequency scale and hence, uniformly spaced points on the unit circle in the $\hat{z}$-plane are mapped onto nonuniformly-spaced points on the unit circle in the $z$-plane.

Applying the mapping of (5) to

$$
X(z)=\sum_{n=0}^{N-1} x[n] z^{-n}
$$

we obtain

$$
X(\hat{z})=\sum_{n=0}^{N-1} x[n] B(\hat{z})^{n}
$$

If we denote

$$
B(\hat{z})=\frac{A(\hat{z})}{\tilde{A}(\hat{z})}
$$

${ }^{1}$ Since the transformation function is required to map the unit circle in $z$-plane to the unit circle in $\hat{z}$-plane, it follows that the mapping must be an allpass function. where $\tilde{A}(\hat{z})$ is the mirror-image polynomial of $A(\hat{z})$, i.e., $\tilde{A}(\hat{z})=\hat{z}^{-M} A\left(\hat{z}^{-1}\right)$, we arrive at

$$
X(\hat{z})=\sum_{n=0}^{N-1} x[n]\left[\frac{A(\hat{z})}{\tilde{A}(\hat{z})}\right]^{n}=\frac{P_{e}(\hat{z})}{D_{e}(\hat{z})} .
$$

Here, the numerator

$$
P_{e}(\hat{z})=\sum_{n=0}^{N-1} x[n] \tilde{A}^{N-1-n}(\hat{z}) A^{n}(\hat{z})
$$

is a polynomial of degree $M(N-1)$ that is a function of $x[n]$. The denominator

$$
D_{e}(\hat{z})=\tilde{A}^{N-1}(\hat{z})
$$

is another polynomial of degree $M(N-1)$ that is, however, not a function of $x[n]$.

WDFT is defined as $X(\hat{z})$ evaluated at $\hat{z}=e^{j 2 \pi k / N}$

$$
\hat{X}[k]=\left.X(\hat{z})\right|_{\hat{z}=e^{j 2 \pi k / N}}=\frac{\left.P_{e}(\hat{z})\right|_{\hat{z}=e^{j 2 \pi k / N}}}{\left.D_{e}(\hat{z})\right|_{\hat{z}=e^{j 2 \pi k / N}}} .
$$

Define

$$
\begin{aligned}
P(\hat{z}) & =P_{e}(\hat{z}) \bmod \hat{z}^{N} \\
& =\sum_{n=0}^{N-1}\left[\sum_{m \geq 0, n+m N \leq M(N-1)} p_{e_{n+m N}}\right] \hat{z}^{-n}
\end{aligned}
$$

where $p_{e_{i}}$ is the $i$ th coefficient of $P_{e}(\hat{z})$. Similarly, define

$$
D(\hat{z})=D_{e}(\hat{z}) \bmod \hat{z}^{N}
$$

$P(\hat{z})$ and $D(\hat{z})$ both have degree $N-1$. Since

$$
\left.\hat{z}^{N}\right|_{\hat{z}=e^{j 2 \pi k / N}}=1
$$

the WDFT computation as shown in (12) may be simplified to

$$
\hat{X}[k]=\frac{\left.P(\hat{z})\right|_{\hat{z}=e^{j 2 \pi k / N}}}{\left.D(\hat{z})\right|_{\hat{z}=e^{j 2 \pi k / N}}}
$$

Let $\hat{P}[k], \hat{D}[k]$ be the $N$ point DFT of the length $N$ sequences obtained from the coefficients of $P(\hat{z})$ and $D(\hat{z})$

$$
\hat{P}[k]=\left.P(\hat{z})\right|_{\hat{z}=e^{j 2 \pi k / N}}, \quad \hat{D}[k]=\left.D(\hat{z})\right|_{\hat{z}=e^{j 2 \pi k / N}}
$$

Then

$$
\hat{X}[k]=\frac{\hat{P}[k]}{\hat{D}[k]} .
$$

Using matrix notation, define the input vector as

$$
\mathrm{x}=\left[\begin{array}{c}
x[0] \\
x[1] \\
\vdots \\
x[N-1]
\end{array}\right]
$$




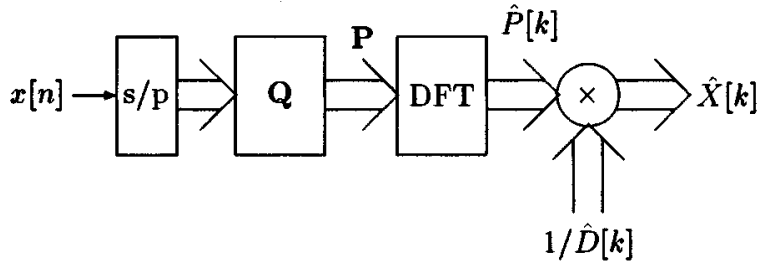

Fig. 2. The proposed warped-DFT realization.

and let $\mathbf{P}$ be the column vector formed from the coefficients of $P(\hat{z})$. Then we can find $\mathbf{P}$ as

$$
\mathbf{P}=\mathbf{Q} \cdot \mathbf{x}
$$

where $\mathbf{Q}$ is an $N \times N$ real matrix discussed in the next section. Further, $\hat{P}[k]$ may be obtained as

$$
\left[\begin{array}{c}
\hat{P}[0] \\
\hat{P}[1] \\
\vdots \\
\hat{P}[N-1]
\end{array}\right]=\mathbf{W} \cdot \mathbf{Q} \cdot \mathbf{x}
$$

where $\mathbf{W}$ is the $N \times N$ DFT matrix, $W=\left\{W_{N}^{i j}\right\}_{0 \leq i, j \leq N-1}$. Finally, the WDFT coefficients are obtained as follows:

$$
\left[\begin{array}{c}
\hat{X}[0] \\
\hat{X}[1] \\
\vdots \\
\hat{X}[N-1]
\end{array}\right]=\Lambda_{D} \cdot \mathbf{W} \cdot \mathbf{Q} \cdot \mathbf{x}
$$

where

$$
\Lambda_{D}=\left[\begin{array}{cccc}
\frac{1}{\hat{D}[0]} & 0 & \cdots & 0 \\
0 & \frac{1}{\hat{D}[1]} & \cdots & 0 \\
\vdots & \vdots & & \vdots \\
0 & 0 & \cdots & \frac{1}{\hat{D}[N-1]}
\end{array}\right] .
$$

Thus, we obtain a factorization of the WDFT matrix into the product of a diagonal matrix, the DFT matrix, and a real matrix. The proposed realization of WDFT, which follows directly from (22), is shown in Fig. 2. The computational advantage of this factorization is discussed later. Contrary to Oppenheim and Johnson's approach, this method is an exact computation of the warped DFT.

Goertzel's algorithm [3] is a popular approach to compute a few samples of DFT. Since in the case of WDFT, a frequency sample at $\hat{\omega}$ no longer satisfies $e^{j \hat{\omega} k / N}=1$, Goertzel's algorithm is not directly applicable to WDFT computation. However, the input sequence may be time-reversed and the Goertzel's algorithm may be applied as given by

$$
y_{k}[n]=x[N-1-n]+e^{-j \hat{\omega}} y_{k}[n-1]
$$

with $y_{k}[-1]=0$ and $\hat{X}[k]=y_{k}[N-1]$, where $\omega=2 k \pi / N$ maps to $\hat{\omega}$ following the transformation of $(5)^{2}$.

WDFT satisfies some, but not all, of the properties of DFT. Linearity and periodicity properties, of course, hold for WDFT. Let $z_{k}, 0 \leq k \leq N-1$, denote the exact points on the $z$-plane

${ }^{2}$ Examples of such mappings are provided in Section III.
TABLE I

SOME PROPERTIES OF WDFT

\begin{tabular}{l|l|l}
\hline Property & Function & WDFT \\
\hline Conjugate symmetry & $x[n]$ real & $\hat{X}[N-k]=\hat{X}^{*}[k]$ \\
Flipping & $x[N-1-n]$ & $z_{k}^{1-N} \hat{X}[N-k]$ \\
Conjugation & $x^{*}[n]$ & $\hat{X}^{*}[N-k]$ \\
\hline
\end{tabular}

where WDFT is evaluated. Let $\hat{z}_{k}$ denote the corresponding points in the $\hat{z}$-plane. Then, $\hat{z}_{N-k}=\hat{z}_{k}^{-1}=\hat{z}_{k}^{*}$. It follows that

$$
z_{N-k}^{-1}=\frac{A\left(\hat{z}_{N-k}\right)}{\tilde{A}\left(\hat{z}_{N-k}\right)}=\frac{\hat{z}_{k}^{M} \tilde{A}\left(\hat{z}_{k}\right)}{\hat{z}_{k}^{M} A\left(\hat{z}_{k}\right)}=z_{k} .
$$

A few properties implied by the above result are shown in Table I.

\section{B. Structure of $\mathbf{Q}$}

The matrix $\mathbf{Q}$ has real coefficients since $A(\hat{z})$ has real coefficients. Since $p_{e_{i}}$ denotes the coefficient of $P_{e}(\hat{z})$, we can write

$$
\left[\begin{array}{c}
p_{e_{0}} \\
p_{e_{1}} \\
\vdots \\
p_{e_{M(N-1)}}
\end{array}\right]=\mathbf{Q}_{e} \cdot \mathbf{x}
$$

where $\mathbf{Q}_{e}$ is an $(M(N-1)+1) \times N$ real matrix. We first show that the rows of $\mathbf{Q}_{e}$ form mirror image pairs. From (10), the $i$ th column of $\mathbf{Q}_{e}$ is obtained from the coefficients of $\tilde{A}^{N-1-i}(\hat{z}) A^{i}(\hat{z})$, whereas the $(N-1-i)$ th column of $\mathbf{Q}_{e}$ is obtained from the coefficients of $\tilde{A}^{i}(\hat{z}) A^{N-1-i}(\hat{z})$. From $\tilde{A}(\hat{z})=\hat{z}^{-M} A\left(\hat{z}^{-1}\right)$ it may be shown that

$$
\tilde{A}^{i}(\hat{z}) A^{N-1-i}(\hat{z})=\hat{z}^{-M(N-1)} \tilde{A}^{N-1-i}\left(\hat{z}^{-1}\right) A^{i}\left(\hat{z}^{-1}\right) .
$$

Since each column is of dimension $M(N-1)$, it follows that the $i$ th column of $\mathbf{Q}_{e}$ is the mirror image of its $(N-1-i)$ th column. Therefore, the rows of $\mathbf{Q}_{e}$ are mirror image pairs as well. If $\mathbf{r}_{e_{j}}$ denotes the $j$ th row of $\mathbf{Q}_{e}$, then $\mathbf{r}_{e_{j}}$ is the mirror image of $\mathbf{r}_{e_{M(N-1)-j}}$.

$\mathbf{Q}$ is obtained from $\mathbf{Q}_{e}$ by adding the rows of the latter, as may be seen from (13). Let $\mathbf{r}_{j}$ denote the $j$ th row of $\mathbf{Q}, 0 \leq$ $j \leq N-1$. Then

$$
\mathbf{r}_{j}=\sum_{k \bmod N=j, 0 \leq k \leq M(N-1)} \mathrm{r}_{e_{k}} .
$$

For an appropriate integer $l$ such that $0 \leq l N-M-j \leq N-1$, the $(l N-M-j)$ th row of $\mathbf{Q}$ is

$$
\mathrm{r}_{l N-M-j}=\sum_{k \bmod } \mathbf{r}_{N=l N-M-j}=\sum_{k \bmod N=j} \mathbf{r}_{e_{M(N-1)-k}} .
$$

This is because $(M(N-1)-j) \bmod N=l N-M-j$, therefore any $k$ such that $k \bmod N=j$ will also satisfy $(M(N-1)-k)$ $\bmod N=l N-M-j$. But $\mathbf{r}_{e_{M(N-1)-k}}$ is the mirror image of $\mathbf{r}_{e_{k}}$. Therefore, it follows that $\mathbf{r}_{l N-M-j}$ is also the mirror image of $r_{j}$. Note that, if for some $j, j=l N-M-j$, then that row is symmetric. 
TABLE II

COMPARISON OF NUMBER OF OPERATIONS

\begin{tabular}{c|cc|cc}
\hline \multirow{2}{*}{$N$} & \multicolumn{2}{|c|}{ Direct } & \multicolumn{2}{c}{ Proposed } \\
& real & real & real & real \\
& mult & add & mult & add \\
\hline 4 & 64 & 56 & 48 & 56 \\
8 & 256 & 240 & 144 & 200 \\
16 & 1024 & 992 & 448 & 704 \\
\hline
\end{tabular}

Since all rows of $\mathbf{Q}$ are mirror image pairs (or, symmetric), each such pair may be expressed as the sum and the difference of a symmetric and an antisymmetric row vector. Thus, $\mathbf{Q} \cdot \mathbf{x}_{n}$ can be computed using only $N / 2$ multiplications for each row.

\section{Number of Operations}

Let $\mathrm{x}$ be an $N$-dimensional complex input vector. Direct computation of the WDFT coefficients from $\mathrm{x}$ (similar to NDFT) requires multiplying x by an $N \times N$ complex matrix, or $4 N^{2}$ real multiplications and $4 N^{2}-2 N$ real additions (assuming one complex multiplication involves four real multiplications and two real additions, and one complex addition involves two real additions). The proposed realization shown in Fig. 2 requires $N^{2}$ real multiplications and $2 N^{2}$ real additions for finding $\mathbf{Q} \cdot \mathbf{x} . N$ point DFT requires $N / 2 \log _{2} N$ complex multiplications and $N \log _{2} N$ complex additions, which amounts to $2 N \log _{2} N$ real multiplications, and $3 N \log _{2} N$ real additions with the earlier assumption. $4 N$ real multiplications and $2 N$ real additions are required for multiplying with $\Lambda_{D}$. Thus, the total requirement is $N\left(N+2 \log _{2} N+4\right)$ real multiplications and $N\left(2 N+3 \log _{2} N\right)$ real additions. Table II shows the required number of operations for some typical values of $N$.

\section{Computation of Inverse WDFT}

The factorization of the WDFT matrix in (22) may be readily used to obtain an efficient computation of inverse WDFT. Assuming the frequency samples of WDFT to be distinct, the inverse WDFT is given by

$$
\mathbf{x}=\mathbf{Q}^{-1} \cdot \mathbf{W}^{-1} \cdot \Lambda_{D}^{-1} \cdot\left[\begin{array}{c}
\hat{X}[0] \\
\hat{X}[1] \\
\vdots \\
\hat{X}[N-1]
\end{array}\right]
$$

where $\mathbf{W}^{-1}$ is the $N \times N$ IDFT matrix, and $\Lambda_{D}^{-1}$ is a diagonal matrix. Since the rows of $\mathbf{Q}$ are mirror image pairs or symmetric, $\mathbf{Q}^{-1}$ is a matrix with real coefficients whose columns are mirror image pairs or symmetric. With an appropriate column permutation, its rows can be made mirror image pairs or symmetric. Therefore, $\mathbf{Q}^{-1}$ can be written as a $\mathbf{Q}$-like matrix post-multiplied by a permutation matrix. Consequently, the computation of an $N$ point inverse WDFT is equivalent to that of an $N$ point WDFT with an additional permutation involving no multiplication.

In case of the first-order $(M=1)$ allpass WDFT, $\mathbf{Q}_{e}=\mathbf{Q}$. Since the columns of $\mathbf{Q}_{e}$ are mirror image pairs, the rows of $\mathbf{Q}^{-1}$ are also mirror-image pairs, and no further permutation is necessary.

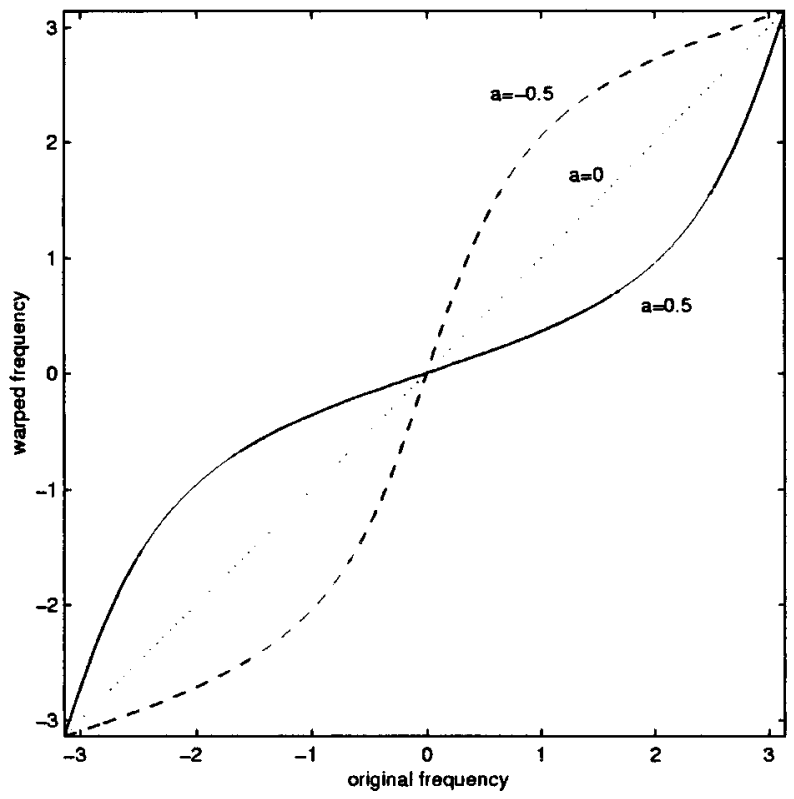

Fig. 3. Frequency mapping for the first-order allpass.

\section{EXAMPLES OF WDFT}

\section{A. First-Order Allpass}

The simplest example of a nontrivial mapping is obtained using a first-order allpass function having $A(\hat{z})=-a+\hat{z}^{-1}$

$$
z^{-1}=\frac{-a+\hat{z}^{-1}}{1-a \hat{z}^{-1}}
$$

where $|a|<1$ for stability [4]. Replacing $z=e^{j \omega}$ and $\hat{z}=$ $e^{j \hat{\omega}}$, where the original angular frequency is $\omega$ and the warped frequency is $\hat{\omega}$, we obtain

$$
e^{-j \omega}=\frac{-a+e^{-j \hat{\omega}}}{1-a e^{-j \hat{\omega}}}=\frac{\left(e^{-j \hat{\omega} / 2}-a e^{j \hat{\omega} / 2}\right)^{2}}{\left|1-a e^{-j \hat{\omega}}\right|^{2}} .
$$

Taking square root of either side, we have

$$
e^{-j \omega / 2}=\frac{e^{-j \hat{\omega} / 2}-a e^{j \hat{\omega} / 2}}{\left|1-a e^{-j \hat{\omega}}\right|}
$$

Taking the ratio of the imaginary part to the real part of each side, the frequency mapping is obtained to be

$$
\tan \left(\frac{\omega}{2}\right)=\left(\frac{1+a}{1-a}\right) \tan \left(\frac{\hat{\omega}}{2}\right)
$$

which is shown in Fig. 3 for some values of $a$.

This transformation retains the ordering of the frequency points. However, for a positive (negative) $a$, the nonlinear mapping stretches the high (low) frequency region providing higher frequency resolution while compressing the remaining part. Conventional DFT becomes a special case when $a=0$ and the mapping is linear. Fig. 4 illustrates the nonuniform sampling of WDFT by showing the location of samples on the unit circle of the $z$ plane for DFT and WDFT for $N=16$. 

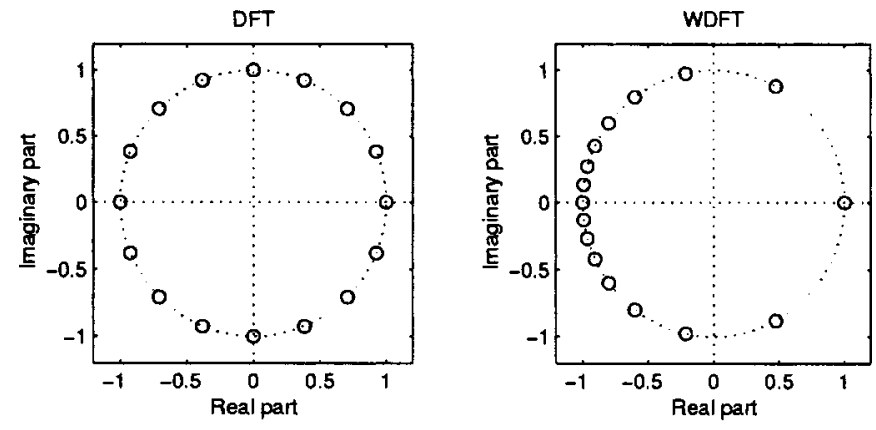

Fig. 4. Location of frequency samples for DFT and WDFT $(a=0.5)$ for $N=16$.

It may be of interest to find the allpass function $A(\hat{z})$ that maps some specific frequency $\omega_{1}$ to some other value $\hat{\omega}_{1}$. For first and second-order case, such solutions are tabulated in [3]. On the other hand, if we wish to obtain a WDFT sample at some specific $\hat{\omega}_{1}$, then there are multiple solutions since $2 k \pi / N$ for any $k$ may be chosen to map to $\hat{\omega}_{1}$. In this case, the solution with the smallest $|a|$ (least warping) may be selected, or some other criterion such as closeness of a sample to another desired frequency $\hat{\omega}_{2}$ may be used.

Since $M=1$, it follows that $P_{e}(\hat{z})=P(\hat{z}), D_{e}(\hat{z})=D(\hat{z})$, and $\mathbf{Q}_{e}=\mathbf{Q}$. The matrix $\mathbf{Q}$ displays the symmetry mentioned in the earlier section. Specifically, $\mathbf{r}_{j}$ and $\mathbf{r}_{N-1-j}$ (for $l=1$ ) are mirror image pairs for all $j$ for even $N$. For odd $N$, all rows except $\mathbf{r}_{(N-1) / 2}$ (which is symmetric) are such mirror image pairs.

Q has, apart from the symmetry, a nice structure. Specifically, the $r s$ th element of $\mathbf{Q}$ is given by

$$
q_{r s}=\sum_{p=\max \{0, r-s\}}^{\min \{r, N-1-s\}}{ }^{N-1-s} C_{p}{ }^{s} C_{r-p}(-a)^{s-r+2 p}
$$

for $0 \leq r, s \leq N-1$, which leads to the following combinatorial rule (similar to those used for similar purposes for bilinear and other transforms [5]). The first row is given by $q_{0 s}=(-a)^{s}, 0 \leq s \leq N-1$, while the first column is given by $q_{r 0}={ }^{N-1} C_{r}(-a)^{r}$. The remaining elements can be found using the recursion relation

$$
q_{r s}=q_{r-1, s-1}-a q_{r, s-1}+a q_{r-1, s} .
$$

This approach is attractive if $\mathbf{Q}$ is to be computed for different values of $a$.

$\mathbf{Q}$ is also amenable to recursive computation. We shall use a subscript in this subsection to denote the size of the warped transform. Thus, $P_{e_{N}}(\hat{z})$ is the numerator polynomial for length $N$ input sequence. If we consider a length $N-1$ input sequence consisting of the first $N-1$ samples $x[0], \ldots, x[N-2]$, then from (10) it follows that

$$
P_{e_{N}}(\hat{z})=P_{e_{N-1}}(\hat{z}) \tilde{A}(\hat{z})+x[N-1] A^{N-1}(\hat{z})
$$

Let $\mathbf{P}_{N}$ be the column vector formed from the coefficients of $P_{e_{N}}(\hat{z})$. For the first-order allpass of (31), the above relation may be expressed in matrix form as

$$
\mathbf{P}_{N}=\mathbf{A}_{N} \cdot \mathbf{P}_{N-1}+\mathbf{B}_{N} \cdot x[N-1]
$$

where $\mathbf{P}_{N-1}$ is the numerator vector computed for the first $N-$ 1 samples $[x[0] x[1] \cdots x[N-2]]^{T} . \mathbf{A}_{N}$ is an $N \times N-1$ sparse Toeplitz matrix,

$$
\mathbf{A}_{N}=\left[\begin{array}{ccccc}
1 & 0 & 0 & \cdots & 0 \\
-a & 1 & 0 & \cdots & 0 \\
0 & -a & 1 & \cdots & 0 \\
\vdots & \vdots & \vdots & \ddots & \vdots \\
0 & 0 & 0 & \cdots & 1 \\
0 & 0 & 0 & \cdots & -a
\end{array}\right]
$$

and $\mathbf{B}_{N}$ is the $N \times 1$ vector formed from the last column of $\mathbf{Q}$

$$
\mathbf{B}_{N}=\left[\begin{array}{c}
{ }^{N-1} C_{0}(-a)^{N-1} \\
{ }^{N-1} C_{1}(-a)^{N-2} \\
{ }^{N-1} C_{2}(-a)^{N-3} \\
\vdots \\
{ }^{N-1} C_{N-1}(-a)^{0}
\end{array}\right] .
$$

The recursion of (38) is useful if more data points are to be added in computing the WDFT for a finer resolution.

Because of symmetry, finding $\mathbf{P}_{N}$ requires $N^{2}$ real multiplications and $2 N^{2}$ real additions, or a total of $3 N^{2}$ operations, for a complex input sequence of length $N$. However, the recursion (38) requires $4(N-1)$ real multiplications and $2(N-2)$ real additions to compute $\mathbf{P}_{N}$ from $\mathbf{P}_{N-1}$. If $\mu_{\text {mul }}(N)$ and $\mu_{\text {add }}(N)$ be the number of real multiplications and real additions needed to compute $\mathbf{P}_{N}$ using this recursion, then

$$
\begin{aligned}
& \mu_{\text {mul }}(N)=4(N-1)+\mu_{\text {mul }}(N-1) \\
& \mu_{\text {add }}(N)=2(N-2)+\mu_{\text {add }}(N-1) .
\end{aligned}
$$

By repeated application of this recursion, we obtain

$$
\mu_{\text {mul }}(N)=2 N(N-1), \quad \mu_{\text {add }}(N)=(N-1)(N-2) .
$$

Thus, it is observed that the number of multiplications nearly doubled using the recursion. However, the total number of operations is $3 N^{2}-5 N+2$, which is marginally smaller than that of the single stage computation.

\section{B. Second-Order Allpass}

While a monotonic mapping is obtained using a first-order allpass, nonmonotonic (multiple) mapping is obtainable using a 


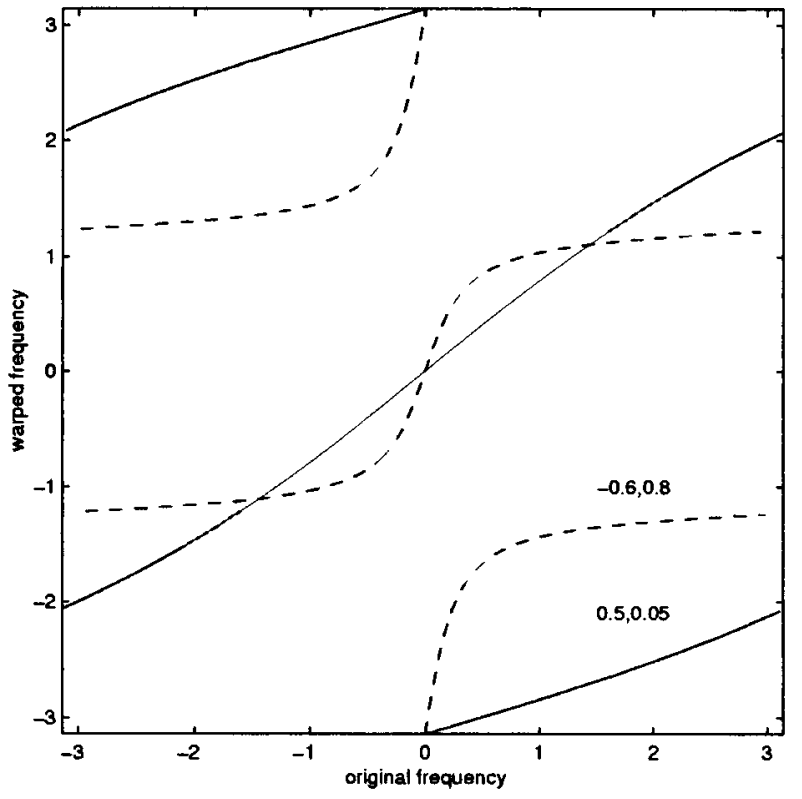

Fig. 5. Frequency mapping for the second-order allpass.

second-order allpass function ${ }^{3}$. We consider $A(\hat{z})=b+a \hat{z}^{-1}+$ $\hat{z}^{-2}$ with real coefficients, such that the mapping is given by

$$
z^{-1}=\frac{b+a \hat{z}^{-1}+\hat{z}^{-2}}{1+a \hat{z}^{-1}+b \hat{z}^{-2}}
$$

where $a$ and $b$ are suitably chosen. Replacing $z=e^{j \omega}$ and $\hat{z}=e^{j \hat{\omega}}$, and after some algebraic manipulations similar to the earlier subsection, we obtain

$$
e^{-j \omega / 2}=\frac{e^{-j \hat{\omega}}+a+b e^{j \hat{\omega}}}{\mid 1+a e^{-j \hat{\omega}}+b e^{-j 2 \hat{\omega} \mid}}
$$

Consequently, the frequency mapping is obtained to be

$$
\cot \left(\frac{\omega}{2}\right)=\left(\frac{1+b}{1-b}\right) \cot \hat{\omega}+\left(\frac{a}{1-b}\right) \operatorname{cosec} \hat{\omega}
$$

which is shown in Fig. 5 for some choices of $a, b$.

The symmetry observed in $\mathbf{Q}$ is as follows. Since the top $N-$ 1 rows of $\mathbf{Q}$ are obtained by adding two rows of $\mathbf{Q}_{e}$, these rows have mirror image pairs $\mathbf{r}_{j}$ and $\mathbf{r}_{N-2-j}$ (for even $N, \mathbf{r}_{(N / 2)-1}$ will be symmetric). The last row, $\mathbf{r}_{N-1}$, equals $\mathbf{r}_{e_{N-1}}$, and is symmetric.

\section{APPLICATIONS OF WDFT}

\section{A. Signal Analysis}

Since WDFT offers the choice to increase frequency resolution at any selected part of the spectral axis without changing $N$, as well as to determine a frequency sample at any exact point by choosing the allpass coefficients, it may be used as an useful

${ }^{3}$ Due to many-to-one mapping, second- (and higher) order allpass may map two distinct frequencies, $\hat{\omega}=2 k_{1} \pi / N$ and $2 k_{2} \pi / N$, to a single frequency $\omega$ (singular WDFT matrix). While such a possibility is unlikely for odd $N$, even $N$ should be avoided since for even $N$ two frequencies $0\left(k_{1}=0\right)$ and $\pi$ $\left(k_{2}=N / 2\right)$ are mapped to $\omega=0$.
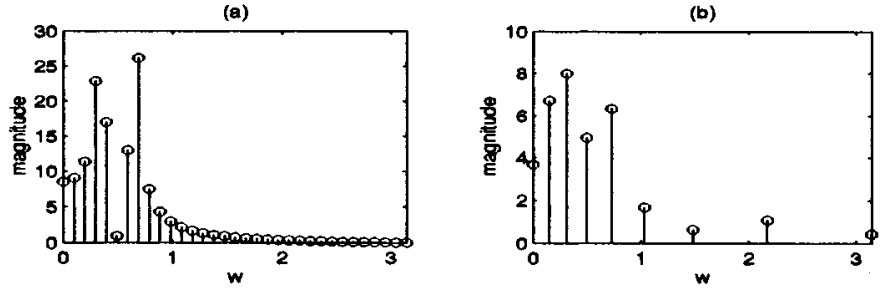

Fig. 6. Magnitude spectrum for two closely-spaced tones. (a) 64-point DFT. (b) 16-point WDFT.

tool in signal analysis. An application of WDFT in signal analysis is thus in resolving two or more closely spaced sinusoids with a shorter length transform than that needed in using the conventional DFT. For example, assuming a transform length that is only an integer power of two, as indicated in Fig. 6(a) a 64-point DFT is required to resolve two sinusoids located at 0.35 and 0.65 radians, while a 16-point WDFT with $a=-0.45$ resolves clearly the two sinusoids as illustrated in Fig. 6(b). Implementation of the (real) 16-point WDFT requires a total of 272 real multiplications. On the other hand, a 64-point FFT requires a total of 704 real multiplications.

\section{B. Tunable FIR Filter Design}

Allpass function-based spectral transformation is used in designing IIR filters from a prototype IIR filter. Using WDFT, this technique is readily extensible to using spectral transformation to design an FIR filter from an FIR prototype. Given an FIR prototype $G(z)$, another FIR filter $\hat{G}(z)$ with a desired type and desired passband edge/edges may be obtained. The form of the allpass function has to be chosen based on the desired type. The coefficients of the allpass function should be chosen such that the passband edge of $G(z)$ maps to the desired passband edge of $\hat{G}(z)$. Let $g[n]$ be the impulse response of the prototype $G(z) . \hat{G}[k]$, the WDFT of $g[n]$, is determined with the chosen allpass function. Now inverse DFT of $\hat{G}[k]$ is computed to obtain the FIR impulse response $\hat{g}[n]$ of the same length yielding the desired filter.

Since for a real sequence, the WDFT coefficients show conjugate symmetry, $\hat{G}[k]$ is conjugate symmetric and $\hat{g}[n]$ is real. However, if the prototype FIR $g[n]$ is linear phase, the obtained FIR $\hat{g}[n]$ will no longer be linear phase. If a linear phase FIR is desirable, the phase response of $\hat{G}[k]$ has to be made linear before taking the IDFT. This is achieved as follows. Define a new warped spectrum $\hat{G}_{l p}[k]$ such that

$$
\begin{aligned}
\left|\hat{G}_{l_{p}}[k]\right| & =|\hat{G}[k]| \\
\operatorname{angle}\left(\hat{G}_{l_{p}}[k]\right) & =-k \frac{(N-1) \pi}{N}, \quad 0 \leq k \leq N-1(46)
\end{aligned}
$$

where $N$ is the length of the prototype FIR $g[n]$. Now IDFT of $\hat{G}_{l p}[k]$ instead of $\hat{G}[k]$ is taken to obtain the desired impulse response. $\hat{G}_{l_{p}}[k]$ obtained from the above equation is conjugate symmetric for odd $N$. However, for even $N$, conjugate symmetry has to be imposed. Note that

$$
\operatorname{angle}\left(\hat{G}_{l p}[k]\right)+\operatorname{angle}\left(\hat{G}_{l_{p}}[N-k]\right)=-(N-1) \pi \neq 0
$$

for even $N$. So, a phase correction term of $\pi$ is added to angle $\left(\hat{G}_{l p}[k]\right)$ for $N / 2+1 \leq k \leq N-1$ when $N$ is even. 


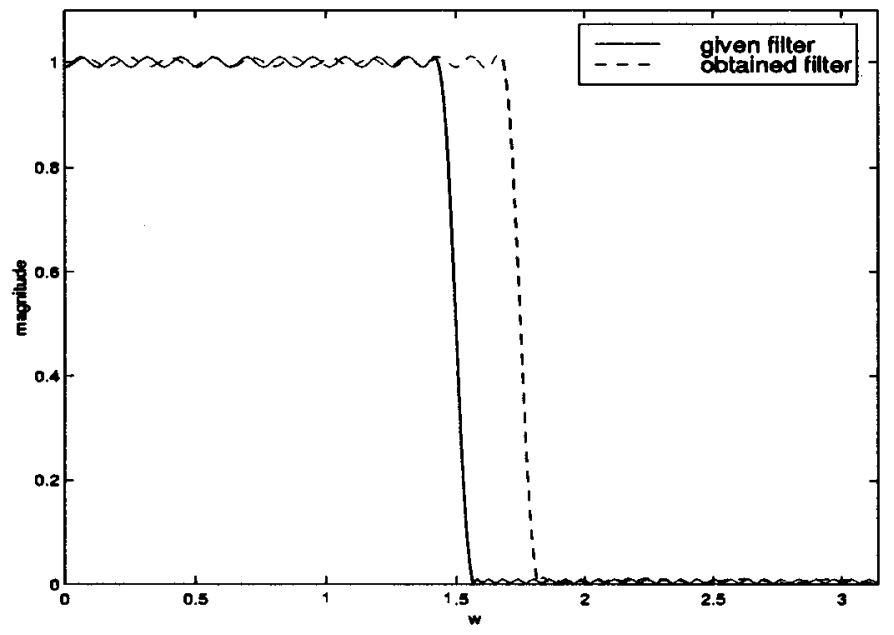

Fig. 7. Magnitude response of the given and the obtained lowpass filters.

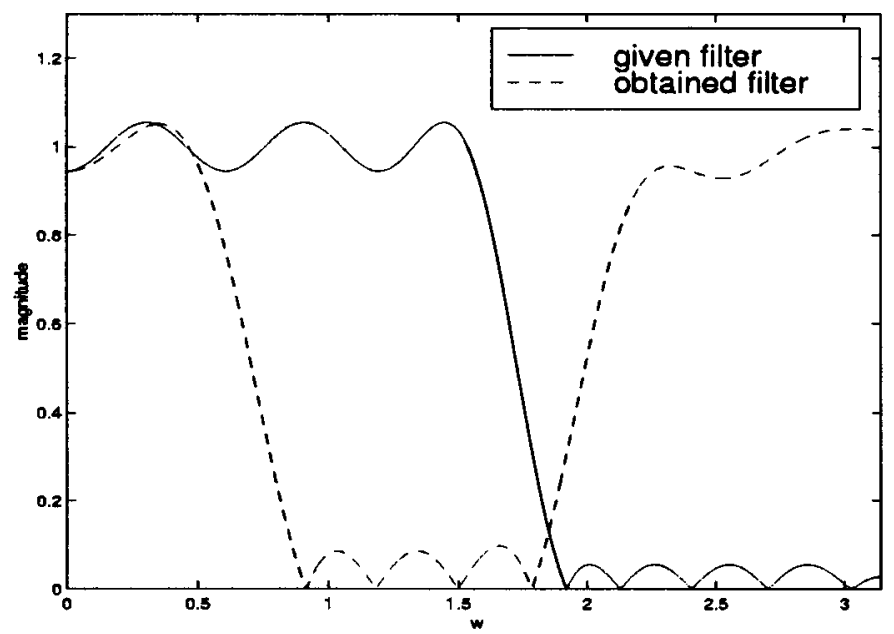

Fig. 8. Magnitude response of the given lowpass and the obtained bandstop filters.

The above technique produces comparable passband and stopband errors for small warping. At large warping, due to the time-domain aliasing the passband/stopband errors become larger than that of the prototype filter. We illustrate the technique using the following two examples.

For a lowpass to lowpass transformation, the first-order allpass function of Section III-A is required. Fig. 7 shows the magnitude response of a prototype lowpass filter with passband extending upto $1.5 \mathrm{rad}$. If it is desirable to map this passband to $1.75 \mathrm{rad}$, then from (34), the required value of $a$ is obtained as 0.1249 . The magnitude response of the obtained filter is also shown in the same figure. In this case, linear phase is not imposed on the desired filter. Note that lowpass-to-highpass transformation is also possible with first-order allpass functions.

For a lowpass-to-bandstop transformation, the second-order allpass function of Section III-B is used. Fig. 8 shows the magnitude response of the prototype lowpass filter and the obtained bandstop filter. The allpass function for this example has $a=$ $-0.3, b=0$. In this example, the prototype length is 21 , and linear phase is imposed on the desired filter. Lowpass-to-bandpass transformation is also possible with second-order allpass functions.

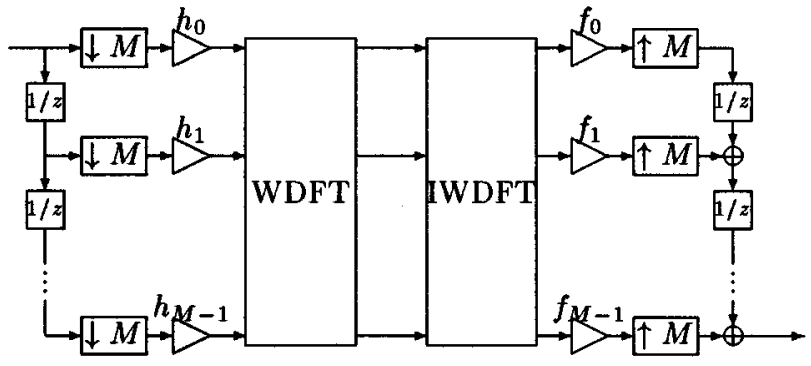

Fig. 9. Warped WDFT-based filterbank.

\section{Warped Filterbank Design}

In this section, it is demonstrated that WDFT may be used to warp a filterbank such that the center frequencies of the bands are spaced nonuniformly. Let

$$
H(z)=\sum_{m=0}^{M-1} A_{m}\left(z^{M}\right) z^{-m}
$$

be a prototype lowpass filter with polyphase components $A_{m}(z)$. This filter is modulated by $e^{j \omega_{k}}$ to get $M$ analysis filters

$$
H_{k}(z)=H\left(z e^{j \omega_{k}}\right)=\sum_{m=0}^{M-1} A_{m}\left(z^{M} e^{j M \omega_{k}}\right) z^{-m} e^{-j m \omega_{k}}
$$

for $0 \leq k \leq M-1$. For the choice of uniform center frequencies, $\omega_{k}=2 k \pi / M$, the polyphase components remain the same, $A_{m}\left(z e^{j M \omega_{k}}\right)=A_{m}(z)$. Consequently, the filterbank of (49) may be realized using a delay chain, followed by a diagonal matrix $\Lambda_{A}$ (whose diagonal elements are $A_{m}(z)$ ), and a transform matrix corresponding to $\left\{e^{-j m \omega_{k}}\right\}$. Further, the transform is IDFT. As a result, the $M$-band uniform DFT filterbank is obtained.

If we choose $\omega_{k}$ according to some allpass warped frequency axis, then the transform is WDFT (with an inverse warping), say $\mathbf{D}_{N}$. However, since $\omega_{k}$ are arbitrary, the polyphase components are indeed modified, unless they are zeroth order, $A_{m}(z)=h_{m}$. For the zeroth-order polyphase case (prototype is an FIR of length $M$ ), the delay chain- $\Lambda_{A}$-transform matrix realization is feasible, and such a filterbank is shown in Fig. 9. From (49), it is seen that the decimation/interpolation factors do not change. The synthesis bank, shown in the above scheme, achieves perfect reconstruction if we choose $f_{m}=1 / h_{m}, 0 \leq m \leq M-1$. This is because $\Lambda_{A}^{-1} \mathbf{D}_{N}^{-1} \mathbf{D}_{N} \Lambda_{A}$ is an identity operation, and the delay chain with decimators and interpolators is PR. In other designs of warped filterbank from a uniform DFT filterbank using allpass transformation [6], [7], the PR property is not retained.

Fig. 10 shows the magnitude response of the analysis filterbank for $M=16, h_{m}=0.25$ for all $m$, and for the allpass coefficient $a=0$ (no warping, uniform filterbank), 0.5 and -0.5 respectively. Due to warping, the passbands are nonuniformly spaced in frequency. This may lead to better objective or subjective compression than the uniform filterbank. For example, the theoretical subband coding gain (ratio of input variance to geometric mean of the subband variances scaled by synthesis 
(a)

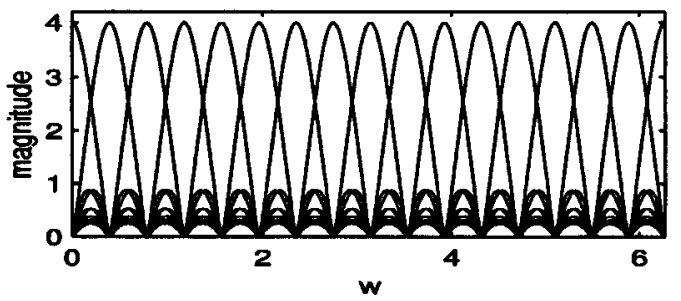

(b)

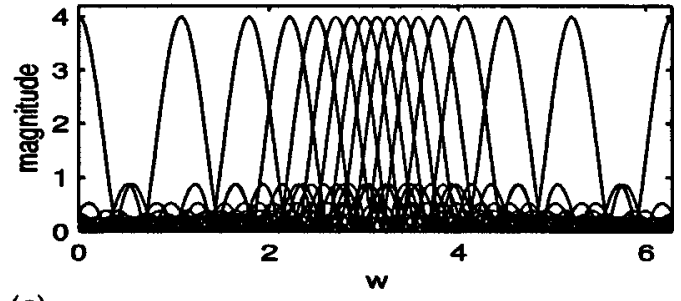

(c)

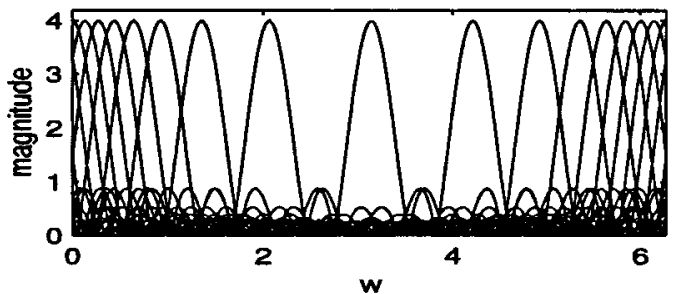

Fig. 10. 16-Band warped filterbank: (a) $a=0$; (b) $a=0.5$; (c) $a=-0.5$.

filter norms) for an AR(1) source with correlation coefficient 0.9 for the filterbank of Fig. 10 is found to be 3.72 using $a=0$ (uniform), and 3.79 using $a=-0.02$ (warped).

\section{CONCLUSION}

In this paper, we discuss the WDFT, which uses allpass transformation to provide nonuniformly spaced frequency samples. By factorizing the WDFT matrix, we have proposed a realization of WDFT which is exact and efficient. We have discussed various properties of WDFT and of the $\mathbf{Q}$ matrix associated with it. First-order and second-order allpass examples are also shown. Finally, a number of applications of WDFT in signal analysis, tunable FIR filter design, and warped filterbank design are also illustrated. We believe that the added flexibility of WDFT in choosing the frequency samples will find applications in many more areas. The WDFT concept has also been extended to the development of warped DCT and applied to image compression [8].

\section{REFERENCES}

[1] A. Oppenheim and D. Johnson, "Computation of spectra with unequal resolution using the fast Fourier transform," Proc. IEEE, vol. 59, pp. 299-301, 1971.

[2] S. Bagchi and S. K. Mitra, Nonuniform Discrete Fourier Transform and its Signal Processing Applications. Norwell, MA: Kluwer, 1999.

[3] S. K. Mitra, Digital Signal Processing: A Computer-Based Approach, 2nd ed. New York: McGraw-Hill, 2001.

[4] A. C. Constantinides, "Spectral transformations for digital filters," Proc. Inst. Elect. Eng., vol. 117, no. 8, pp. 1585-1590, 1970.

[5] E. I. Jury and O. W. C. Chan, "Combinatorial rules for some useful transformations," IEEE Trans. Circuit Theory, vol. CT-20, pp. 476-480, Sept. 1973.

[6] T. Gulzow, A. Engelsberg, and U. Heute, "Comparison of a discrete wavelet transform and a nonuniform polyphase filterbank applied to spectral-subtraction speech enhancement," Signal Processing, vol. 64 , pp. 5-19, 1998.

[7] M. Kappelan, B. Straub, and P. Vary, "Flexible nonuniform filter banks using allpass transformation of multiple order," in Proc. European Signal Processing Conf. EUSIPCO'96, vol. III, 1996, pp. $1745-1748$.

[8] N. I. Cho and S. K. Mitra, "Warped discrete cosine transform and its application in image compression," IEEE Trans. Circuits Syst. Video Technol, vol. 10, pp. 1364-1373, Dec. 2000.

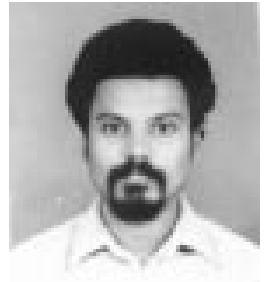

Anamitra Makur (M'00-SM'00) received the B.Tech. degree in electronics and electrical communication engineering from the Indian Institute of Technology, Kharagpur, India, in 1985 and the M.S. and $\mathrm{Ph} . \mathrm{D}$. degrees in electrical engineering from the California Institute of Technology, Pasadena, in 1986 and 1990.

He is currently an Associate Professor of electrical communication engineering, Indian Institute of Science, Bangalore, India. His research interests in signal compression include subband coding, filterbank design and multirate DSP, vector quantization, motion field coding, other image/video compression schemes and standards, and multimedia applications. His interests in image/video processing includes watermarking, halftoning, restoration, segmentation, and multidimensional DSP.

Dr. Makur is the recipient of the 1998 Young Engineer Award from the Indian National Academy of Engineering.

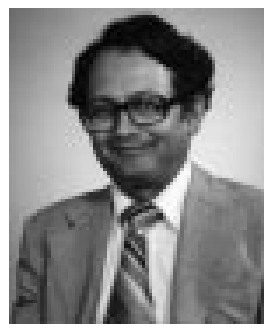

Sanjit K. Mitra (S'59-M'63-SM'69-F'74-LF'01) received the B.Sc. (Hons.) degree in physics in 1953 from Utkal University, Cuttack, India, the M.Sc. (Tech.) degree in radio physics and electronics in 1956 from Calcutta University; the M.S. and Ph.D. degrees in electrical engineering from the University of California at Berkeley, in 1960 and 1962, respectively and an Honorary Doctorate of Technology degree from the Tampere University of Technology, Finland in 1987.

From June 1962 to June 1965, he was an Assistant Professor of Electrical Engineering at the Cornell University, Ithaca, NY. From June 1965 to January 1967, he was with the AT\&T Bell Laboratories, Holmdel, NJ. He has been on the faculty at the University of California since then, first at the Davis campus and at the Santa Barbara campus since 1977, where he is now a Professor of Electrical and Computer Engineering, and served as Chairman of the Department from July 1979 to June 1982 . He served as the President of the IEEE Circuits and Systems Society in 1986 and as a Member-at-Large of the Board of Governors of the IEEE Signal Processing Society from 1996-1999. He is currently a member of the editorial boards of four journals. He has published over 500 papers in signal and image processing, twelve books and holds five patents.

Dr. Mitra is the recipient of the 1973 F.E. Terman Award and the 1985 AT\&T Foundation Award of the American Society of Engineering Education, the Education Award of the IEEE Circuits and Systems Society in 1989, the Distinguished Senior U.S. Scientist Award from the Alexander von Humboldt Foundation of Germany in 1989, the Technical Achievement Award of the IEEE Signal Processing Society in 1996, the Mac Van Valkenburg Society Award and the CAS Golden Jubilee Medal of the IEEE Circuits and Systems Society in 2000, and the IEEE Millennium Medal in 2000. He is the co-recipient of the 2000 Blumlein-Browne-Willans Premium of the the Institution of Electrical Engineers (London). He is an Academician of the Academy of Finland. He is a Fellow of the AAAS and SPIE, and a member of EURASIP and ASEE. 